\title{
An examination of separation anxiety disorder symptoms in adults from a clinical population
}

\author{
William W Hale III*, Berg A and Malhotra S \\ Utrecht University, Research Center Adolescent Development, 3508 TC Utrecht, The Netherlands
}

\begin{abstract}
Objective: Since major research has only recently begun into adult SepAD in clinical populations, this study examined the phenomenology of patients at-risk for adult SepAD.

Method: A sample of 95 adult patients receiving CBT for either mood or anxiety disorders at a mental health outpatient clinic were included in this study.

Results: It was found that one-fifth of the patients were at-risk for adult SepAD at the start of therapy. The large majority of the at-risk patients were female, were diagnosed at intake with another anxiety disorder, had a diagnosed DSM-IV-TR Axis-IV psychosocial and environmental problem. It was also found that adult SepAD symptom severity decreased after CBT. Lastly, it was found that adult SepAD symptom severity at the start of therapy reduced the effectiveness of CBT for depression, but not for anxiety.
\end{abstract}

Conclusion: The findings may provide a better insight into phenomenology and possible improvement of the treatment of adult SepAD.

\section{Introduction}

The relatively recent inclusion of an adult variant of Separation Anxiety Disorder (SepAD) in the DSM-5 [1] is based on clinician reports of encountering separation anxiety in adult patients seeking treatment for another anxiety disorders [2], as well as ongoing research in clinical and community samples [3]. A recent study by Silove and colleagues [4] using the World Mental Health Survey dataset found the lifetime prevalence rate of adult SepAD to be $5 \%$ across all countries. In clinical samples, $20-23 \%$ of outpatients with anxiety or mood disorders have been found to qualify for an adult SepAD diagnosis $[5,6]$. With respect to the role of gender, a literature study found that in childhood as well as adulthood, more females suffer from the disorder than males [7].

In a clinical sample of patients with anxiety or mood disorders, those with adult SepAD reported to have significantly lower psychosocial functioning in areas of work, home management, close relationships and leisure activities than those without adult SepAD [6]. Further, those diagnosed with SepAD for the first time in adulthood display greater impairment in family and life activities than those whose SepAD persisted from childhood into adulthood [5].

\section{Why hasn't adult SepAD previously received more study?}

The question then arises, why has adult SepAD received such little research attention in the past? Manicavasagar and Silove [8] suggest reasons such as unfamiliarity with adult SepAD among clinicians. This is in part due to previous versions of the DSM, such as the DSM-IV-TR (American Psychiatric Association, 2000), listed SepAD in the section entitled "Disorders Usually First Diagnosed in Infancy, Childhood, or Adolescence" that had the criterion that the disorder had an onset before the age of 18, which precluded an adult onset diagnosis. Moreover, symptoms of adult SepAD overlap with other anxiety disorders, such as panic disorder $[9,10]$, and mood disorders, such as major depression $[11,12]$, making them hard to distinguish from one another.

Adult SepAD overlaps most with panic disorder, so much so that Klein [13] proposed a separation anxiety - panic disorder hypothesis suggesting that childhood SepAD may show heterotypic continuity by developing into separate types of panic disorder in adults. Panic disorder has been found to be the most comorbid disorder with adult SepAD [6] and in fact, the only anxiety disorder significantly related to adult SepAD in one study with a mixed community and clinical sample [14].

Researchers studying adult SepAD have tried to bring out these differences to make a case for the recognition of the disorder as being a distinct, separate category of psychopathology. This is particularly important to do, given that a misdiagnosis could potentially mean a poor prognosis for the afflicted individual. However, since adult SepAD has not received the same level of empirical research as many other DSM disorders clinicians may feel uncomfortable diagnosing an individual with a disorder about which they are unfamiliar with from the literature [14].

\section{Summary and the current study}

It is due to this lack of study into the phenomenology of adult SepAD, that the first goal was to examine the characteristics of those

${ }^{\star}$ Correspondence to: William W. Hale III, Utrecht University, Research Center Adolescent Development, 3508 TC Utrecht, The Netherlands, E-mail: B.Hale@uu.nl

Key words: anxiety, cognitive behavior therapy, depression, outpatient clinic, separation anxiety disorder

Received: April 23, 2018; Accepted: May 18, 2018; Published: May 22, 2018 
at-risk for adult SepAD who received treatment for another depressive or anxiety disorder in order to better understand adult SepAD in the clinical setting. Since it is assumed that adult SepAD is many times an outgrowth of child SepAD $[2,3]$, we specifically selected these two broad psychiatric disorder categories since it has been shown in child and adolescent studies that depression and anxiety many times are strongly comorbid with SepAD and one another $[15,16]$ and display similar developmental trajectories with one another for both males and females [17]. Specifically, the comorbidity with DSM-IV-TR Axis-I diagnoses and significant psychosocial and environmental diagnoses (DSM-IV-TR Axis-IV) of persons at-risk for adult SepAD will be examined in order to assess if the findings of this study compare to that of previous studies.

As previously noted, adult SepAD may often remain unrecognized due to symptom similarities with other DSM disorders, which allows it to blend into the background of the predominant diagnosed pathology without knowledge what effects this background adult SepAD might have on the diagnosed and treated psychopathology. Hence, this study also examined what effect adult SepAD symptomology might have on the treatment of the diagnosed pathology from this clinical population by formulating the following two additional study goals. Specifically, the second goal of this archive data study was to examine the (lack of) development of SepAD symptoms in an adult clinical population when treated with Cognitive Behavioral Therapy (CBT) for another anxiety or depressive disorder. This was done to assess if adult SepAD symptomology might be similar enough to either depression or other anxiety disorders that the CBT would also affect development of adult SepAD symptoms from pre-treatment to post-treatment. If adult SepAD is truly independent from the disorder that CBT was focused on, it would stand to reason that while the symptoms of the disorder targeted by CBT would decrease, that the adult SepAD would either remain constant or even increase due to a lack of treatment. In a study by Bögels et al. [18] it was found that patients with comorbid adult SepAD who received CBT for panic disorder, GAD, or social phobia had poorer outcomes than patients without this comorbid diagnosis. It is due to this aforementioned reason that the third goal was to examine whether comorbid adult SepAD symptoms might influence therapy outcome.

It should be noted that the analyses for these second and third goals were conducted for the entire adult sample, as well as separately for the anxiety and the depression samples.

\section{Method}

\section{Participants and procedure}

For the present archive study, a sample of adult patients receiving CBT for either a mood or an anxiety disorder at the university mental health outpatient clinic of the faculty of Social and Behavioral Sciences of Utrecht University (The Netherlands) were included. All patients who were in therapy from August 2006 to May 2017 were selected to participate in the study if they had completed the adult version of the Screen for Child Anxiety and Related Disorders (SCARED) [19] at the start of their therapy. To be included in the study, participants had to have given approval that their data could be used for scientific purposes. This resulted in a total sample of 95 participants, aged between 18 and $59\left(64.2 \%\right.$ female, $\left.M_{\text {age }}=23.76, S D_{\text {age }}=6.71\right)$. Of these 95 participants, 44 had a DSM-IV-TR [20] mood disorder diagnosis and 51 had an anxiety disorder diagnosis at intake. The SCARED and the Beck Depression Inventory-II used in the current study were completed at the start and the end of therapy for diagnostic purposes and as part of the Routine Outcome Monitoring (ROM) of the therapy process. The study was approved by the ethics committee of the faculty of social and behavioral sciences of Utrecht University (The Netherlands).

\section{Questionnaires}

Participants completed two psychological questionnaires at the start (pre-test) and the end (post-test) of therapy to measure anxiety and depressive disorder symptom dimensions.

\section{Anxiety disorder and SepAD symptom dimensions}

Anxiety disorder symptom dimensions were measured with the total score of the Dutch 38-item adult version of the Screen for Child Anxiety Related Emotional Disorders [19]. This questionnaire consists of items that were answered on a 3-point Likert scale ranging from ' $0=$ almost never' to ' $2=$ often'. Item scores were summed into a total score of anxiety symptoms (Cronbach's $\alpha=.874$ at pretest and $\alpha=.928$ at posttest).

The SepAD symptom dimension consists of 8 -items that were summed into a total SepAD scale score (Cronbach's $\alpha=.668$ at pretest and $\alpha=.764$ at posttest). Two example items would be: 'I am afraid to be alone in the house' and 'I don't like to be away from my family'. In a study of the SCARED for an adult population that included four additional items for the adult SepAD symptom dimension scale found the psychometric properties for this questionnaire as well for the SepAD scale to be strong [21].

\section{Depressive symptom dimensions}

Depressive symptom dimensions were measured using the Dutch version of the Beck Depression Inventory-II [22]. This questionnaire consists of 21-items, each consisting of four statements with increasing severity. An example question would be: $0=$ 'I do not feel sad', $1=$ 'I feel sad much of the time', 2 = 'I am sad all the time', and 3 = 'I am so sad or unhappy that I can't stand it'. Participants were asked to indicate for each item which statement best describes how they have been feeling the past two weeks, including the day the participant filled in the questionnaire. Item scores were summed into a total score of depressive symptoms (Cronbach's $\alpha=.895$ at pretest and $\alpha=.949$ at posttest). In a previous study of the questionnaire, the psychometric properties of the BDI-II have been shown to be strong [23].

\section{Questionnaire missing data}

Out of the total questionnaires received, a maximum of $1.04 \%$ of the scale-item values was missing per questionnaire. Little's Missing Completely At random tests (MCAR; [24]) indicated that these missing values were completely at random (SCARED pre-test: $\chi^{2}(111)=118.10$, $p=.305$; SCARED post-test: $\chi^{2}(111)=112.95, p=.431$; BDI pre-test: $\chi^{2}(20)=22.01, p=.340$; BDI post-test: $\left.\chi^{2}(30)=26.25, p=.662\right)$. Since it was assumed that scale-item values were missing at random, these missing values were imputed in IBM SPSS Statistics 24 (SPSS; [25]) per questionnaire using Relative Mean Substitution (RMS; [26]).

\section{Data analysis}

The first goal of this study was to examine the characteristics of patients at-risk for adult SepAD from a clinical population. Patients were identified being at-risk for adult SepAD if their SepAD scale scores were more than one Standard Deviation $(S D)$ higher than the sample mean $(>$ Mean $+1 S D)$, in accordance with the Dutch manual of the SCARED [27]. 
Frequency analyses in SPSS were used to examine the occurrence of individuals at-risk of having adult SepAD in the current sample and the comorbidity of these adult SepAD symptoms with other DSM-IVTR Axis-I anxiety and depressive disorders and Axis-IV problems. A chi-square test of association was used to examine whether at-risk individuals were significantly more common in individuals diagnosed with an anxiety versus a mood disorder at intake.

The second goal of this study was to examine the (lack of) development of adult SepAD symptoms in a clinical population when treated with $\mathrm{CBT}$ for another internalizing disorder. Using a repeatedmeasures ANOVA it was examined whether there was a significant difference in adult SepAD symptom dimension severity between pretest (at the start of therapy) and post-test (after CBT). It was examined whether differences in adult SepAD symptoms from the start to after CBT could be found in both the anxious and depressed samples or in only one of these samples.

Lastly, it was examined whether adult SepAD symptoms might influence therapy outcome. To do this, linear regression analyses were conducted to examine whether adult SepAD symptom severity at therapy start predicted anxiety symptoms (SCARED total score) or depressive symptoms (BDI total score) at the end of therapy, thereby controlling for these symptom scores at the start of therapy. It was examined whether adult SepAD symptom scores were predictive of therapy outcome in both the anxious and depressed samples or in only one of these samples.

The occurrence and comorbidity of adult SepAD symptoms were examined in the whole sample of 95 participants. A part of this sample also completed the other pre- and post-questionnaires and could therefore be used in some more in-depth analyses (repeated measures ANOVAs and linear regression analyses). The exact sample size that was available for these analyses will be reported per analysis in the result section. Participants who completed all questionnaires at the start and the end of therapy (53.7\%) did not differ significantly from those who did not complete all the questionnaires in respect to age, $F(1,91)=0.16, p=.690$, partial $\eta^{2}=.00$. Also, no significant difference in diagnosis (mood or anxiety diagnosis at intake) was found between participants who did and did not complete all the questionnaires, $\chi^{2}(1)$ $=0.02, p=.876$, Cramer's $V=.02$. However, females were more likely to have completed all the questionnaires than males, $\chi^{2}(1)=4.15, p=$ .042 , Cramer's $V=.21$.

\section{Results}

\section{Descriptive statistics}

Mean levels and standard deviations of adult SepAD symptoms, overall anxiety and depressive symptoms at the start and the end of therapy are presented in Table 1. At the start of therapy, adult SepAD symptoms were significantly higher in females than males, $F(1,93)$ $=8.76, p=.004$, partial $\eta^{2}=.09$. It is interesting to note that adult SepAD symptom scores at therapy start were not significantly higher in participants diagnosed with an anxiety disorder than participants diagnosed with a mood disorder, $F(1,93)=1.63, p=.204$, partial $\eta^{2}$ $=.02$.

\section{Phenomenology of adult SepAD in the clinical setting}

In the current sample of 95 depressed and anxious patients, a little more than one-fifth $(22.1 \%)$ were at-risk for adult SepAD at the start of therapy. Of these patients who were at-risk, two-thirds (66.7\%) was female. Additionally, two-thirds $(66.7 \%)$ of the at-risk patients were
Table 1. Descriptive statistics per variable

\begin{tabular}{|l|c|c|c|}
\hline & $\boldsymbol{N}$ & $\boldsymbol{M}$ & $\boldsymbol{S D}$ \\
\hline Adult SepAD start of therapy (SCARED scale) & 95 & 3.01 & 2.66 \\
\hline Males & 34 & 1.97 & 1.96 \\
\hline Females & 61 & 3.59 & 2.83 \\
\hline Adult SepAD end of therapy (SCARED scale) & 56 & 1.43 & 2.12 \\
\hline Anxiety symptoms start of therapy (SCARED) & 95 & 29.28 & 11.05 \\
\hline Anxiety symptoms end of therapy (SCARED) & 56 & 17.71 & 11.51 \\
\hline Depressive symptoms start of therapy (BDI) & 93 & 18.52 & 9.93 \\
\hline Depressive symptoms end of therapy (BDI) & 55 & 10.03 & 10.36 \\
\hline
\end{tabular}

diagnosed at intake with an anxiety disorder, while the remaining third had a mood disorder diagnosis at intake. However, being at-risk for adult SepAD was not significantly more common in individuals diagnosed with an anxiety disorder than in patients with a mood disorder, $\chi^{2}(1)=1.83, p=.176$, Cramer's $V=.14$.

Most of the participants with at-risk adult SepAD scores were diagnosed with Generalized Anxiety Disorder (33.3\%), Major Depressive Disorder (33.3\%), or Panic Disorder (23.8\%). The large majority at-risk for adult SepAD (61.9\%) had an DSM-IV-TR Axis-IV diagnosis of educational problems, while a little more than one-fourth (28.6\%) had no Axis-IV diagnosis. The remaining at-risk individuals suffered from problems related to the social environment $(4.8 \%)$ or other psychosocial and environmental problems (4.7\%).

\section{Change in adult SepAD severity}

To examine the (lack of) development of adult SepAD symptoms for those treated with CBT for another internalizing disorder, repeated measures ANOVA's were used to inspect whether there was a significant difference in adult SepAD symptom severity between the start and the end of therapy. When analyzing the total sample, it was found that adult SepAD symptom severity decreased significantly from the start to the end of CBT, $F(1,55)=23.85, p<.001$, partial $\eta^{2}=.30\left(M_{\text {pre }}=2.95\right.$, $\left.M_{\text {post }}=1.43, N=56\right)$. Furthermore, results showed that adult SepAD symptom severity decreased in the anxiety sample, $F(1,30)=15.00$, $p=.001$, partial $\eta^{2}=.33\left(M_{\text {pre }}=3.32, M_{\text {post }}=1.43\right)$, as well as in the depression sample, $F(1,24) \stackrel{\text { pre }}{=} 10.31, p=.004$, partial $\eta^{2}=.30\left(M_{\text {pre }}=\right.$ $\left.2.48, M_{\text {post }}=1.44\right)$. These results indicate that adult SepAD symptom severity decreases when anxious and depressed adults are treated with CBT.

\section{Adult SepAD as predictor for therapy outcome}

Lastly, it was examined whether adult SepAD symptoms might influence therapy outcome. As can be seen in the upper part of Table 2 , adult SepAD symptom severity at the start of therapy was not a significant predictor of anxiety symptoms or mood symptoms at the end of CBT when analyzing the whole sample together.

We then investigated the sample of anxious individuals. As can be seen in Table 2, adult SepAD symptom severity at the start of therapy did not predict the severity of anxiety symptoms or depressive symptoms at the end of their therapy. These results imply that adult SepAD symptom severity at the start of therapy does not influence therapy outcome for those receiving CBT for their anxiety disorder.

For the depressed sample, adult SepAD symptom severity at the start of therapy did not predict the depressive symptoms at the end of therapy as a measure of therapy outcome. However, the model with anxiety symptoms at the start of therapy as control variable and adult SepAD symptom severity at the start of therapy as predictor, did significantly predict anxiety symptom scores at the end of therapy in 
Table 2. Regression coefficients for separation anxiety symptom severity predicting anxiety symptoms and depressive symptoms at the end of therapy

\begin{tabular}{|l|c|c|c|c|c|c|}
\hline & $\boldsymbol{N}$ & $\boldsymbol{B}$ & $\boldsymbol{S E}$ & $\boldsymbol{\beta}$ & $\boldsymbol{p}$ \\
\hline & Total sample \\
\hline Anxiety symptoms end of therapy (SCARED) & 56 & -0.03 & 0.58 & -.01 & .960 \\
\hline Depressive symptoms end of therapy (BDI) & 54 & -0.31 & 0.50 & -.08 & .539 \\
\hline & Anxious subsample \\
\hline Anxiety symptoms end of therapy (SCARED) & 31 & -0.85 & 0.83 & -.22 & .315 \\
\hline Depressive symptoms end of therapy (BDI) & 28 & -0.70 & 0.59 & -.20 & .249 \\
\hline & Depressed subsample & \\
\hline Anxiety symptoms end of therapy (SCARED) & 25 & 1.40 & 0.61 & .33 & .031 \\
\hline Depressive symptoms end of therapy (BDI) & 26 & 0.34 & 0.86 & .09 & .696 \\
\hline
\end{tabular}

the depression sample, $F(2,22)=18.38, p<.001$. This model explained about $59.2 \%$ of the variance in anxiety symptoms at the end of therapy (adjusted $R^{2}=.59$ ). In other words, higher overall anxiety symptoms and higher adult SepAD scores at the start of therapy for the individuals who were diagnosed with depression were related to higher anxiety symptoms at the end of therapy. This implies that more severe adult SepAD symptoms at the start of therapy might reduce the effectiveness of CBT in decreasing anxiety symptoms in adults receiving CBT for their diagnosed depressive disorder.

\section{Discussion}

As was noted in the introduction, in this study of adult SepAD symptoms in a clinical population had three distinct research goals. The first goal was to examine the phenomenology of being at-risk for adult SepAD when also receiving a treatment for another depressive or anxiety disorder in order to better understand the characteristics of adult SepAD in the clinical setting. The second goal of this study was to examine the (lack of) development of adult SepAD symptoms in a clinical population when treated with Cognitive Behavioral Therapy (CBT) for another internalizing disorder. And, finally, the third goal was to examine whether comorbid adult SepAD symptoms might influence therapy outcome.

In respect to the first goal, it was found that in an adult out-patient population coming for treatment for depressive or anxiety disorders that a little more than a fifth also suffered from adult SepAD. So clearly adult SepAD is more prevalent in adult populations than has been previously assumed. Furthermore, this group is characterized by being predominantly female (two-thirds) and having strong comorbidity to Generalized Anxiety Disorder, Major Depressive Disorder, or Panic Disorder. Finally, the large majority of this group had an DSM-IV-TR Axis-IV diagnosis of study or school problems similar to previous studies $[5,6]$. So why this group at-risk for adult SepAD remained undiagnosed may be partially explained by adult SepAD hiding in plain sight behind other well-known disorders and/or reluctance of clinicians in diagnosing adult SepAD as has been recently postulated by Manicavasagar and Silove [8]. In both cases, clinicians might consider including measures of SepAD symptomology in their standard diagnostic measures of internalizing disorders in order to prevent patients with elevated adult SepAD symptomology from going unrecognized.

In respect to what effect adult SepAD symptomology might have on the treatment of the diagnosed internalizing disorder, two additional goals were formulated. Since adult SepAD symptoms were not specifically being targeted by the CBT it was suggested that while the symptoms of the disorder targeted by CBT would decrease, that the adult SepAD would either remain constant or even increase (due to a lack of treatment). However, the opposite was found; adult SepAD symptom severity decreased after CBT, for the group as a whole as well as the anxious and depressed samples. And, finally, the findings of the third goal demonstrated that adult SepAD symptom severity at the start of therapy does not influence therapy outcome in adults receiving CBT for an internalizing disorder when the research population is viewed as a whole. However, when the sample was split in an anxiety sample and a depression sample, this same finding was true for the anxiety sample, however it was found that adult SepAD symptom severity at the start of therapy seems to reduce the effectiveness of CBT for depression.

Therefore, when the last two goals are viewed together, it would appear that it is possible that symptom overlap [9] between adult SepAD and the other various internalizing disorders might account for these findings. However, the negative effect of adult SepAD symptom severity on CBT for depression effectiveness seems to be at odds with this view, albeit that adult SepAD symptom severity did decrease after CBT for depression. Hence it is conceivable that there is specific consultation of depression symptoms that do not align as closely as anxiety symptoms when compared to adult SepAD. Nevertheless, one should be mindful that symptom overlap does not make it the same phenomenon. For example, while previously some have argued that the symptoms of anxiety and depression are simply different expressions of the same underlying disorder [28], more recent studies have demonstrated that despite symptom overlap that anxiety and depression are two distinct disorders with their own unique (albeit related) characteristics [17]. In order to better understand the specific relationship adult SepAD holds with depression, additional research is clearly needed.

The study has some limitations that could be addressed in future research. Firstly, the overall sample size of the study was relatively small which limits the generalizability of the findings and prevented an investigating the relation between the specific kinds of anxiety and mood disorders and separation anxiety other than the comorbidity percentage findings. Therefore, future studies may be advised to employ large samples with a focus on the specific comorbid anxiety and mood disorders to better understand the epidemiology and etiology of adult SepAD.

Despite these limitations, this study is a pioneering effort in understanding the phenomenology and possible improvement of the treatment of adult SepAD. A very recent SSRI study of patients with the principal diagnosis of adult SepAD exhibits how progress is being made [29]. And as recently noted by Baldwin, Gordon, Abelli, and Pini, [30], "(T)he inclusion of separation anxiety disorder within the DSM5 group of anxiety disorders diagnosable in adults certainly provides impetus for further research into its distinguishing psychopathological phenotypic features ... which might help delineate it from frequent comorbid conditions, such as panic disorder, agoraphobia, and social anxiety disorder." (p. 293). Hence, continued research in this direction with bigger community and clinical samples will not only advance scientific knowledge, but also have a domino effect of then reducing clinician unfamiliarity with the disorder, misdiagnoses and lack of or no treatment of adult SepAD.

\section{Acknowledgments}

We would like to thank the staff of the university mental health outpatient clinic of the faculty of Social and Behavioral Sciences of Utrecht University in their help in conducting this study. This research was conducted at the Research Center Adolescent Development 
department and the university mental health outpatient clinic, both of the faculty of Social and Behavioral Sciences of Utrecht University.

\section{Funding}

This research did not receive any specific grant from funding agencies in the public, commercial, or not-for-profit sectors.

\section{Conflicts of Interest}

None.

\section{References}

1. American Psychiatric Association (2013) Diagnostic and statistical manual of mental disorders. 5th ed, Washington, DC, American Psychiatric Association.

2. Manicavasagar V, Silove D, Curtis J (1997) Separation anxiety in adulthood: A phenomenological investigation. Compr Psychiatry 38: 274-282.

3. Silove D, Manicavasagar V, Drobny J (2002) Associations between juvenile and adult forms of Separation Anxiety Disorder: A study of volunteers with histories of school refusal. J Nerv Ment Dis 190: 413-414.

4. Silove D, Alonso J, Bromet E, Gruber M, Sampson N, Scott K, et al. (2015) Pediatriconset and adult-onset separation anxiety disorder across countries in the world mental health survey. Am J Psychiatry 172: 647-656.

5. Pini S, Abelli M, Shear KM, Cardini A, Lari L, Gesi C, et al. (2010) Frequency and clinical correlates of adult separation anxiety in a sample of 508 outpatients with mood and anxiety disorders. Acta Psychiatrica Scandinavica 122: 40-46.

6. Silove D, Marnane C, Wagner R, Manicavasagar V, Rees S. (2010) The prevalence and correlates of adult Separation Anxiety Disorder in an anxiety clinic. BMC Psychiatry 10: 21-27.

7. Bogels SM, Knappe S, Clark LA. (2013) Adult separation anxiety disorder in DSM-5. Clin Psychol Rev 33: 663-674.

8. Manicavasagar V, Silove D (2016) Why are we still missing adult separation anxiety disorder in clinical practice? Aust NZ J Psychiatry 50: 807-808.

9. Silove D, Marnane C (2013) Overlap of symptom domains of separation anxiety disorder in adulthood with panic disorder-agoraphobia. J Anxiety Disord 27: 92-97.

10. Starcevic, V. (2013). Looking for balance between promoting adult separation anxiety disorder and overlooking it. Aust NZ J Psychiatry 47: 782-784.

11. Carmassi C, Gesi C, Corsi M, Pergentini I, Cremone IM, Conversano C, et al. (2015) Adult separation anxiety differentiates patients with complicated grief and/or major depression and is related to lifetime mood spectrum symptoms. Compr Psychiatry 58: 45-49.

12. Pini S, Abelli M, Mauri M, Muti M, Iazzetta P, Banti S, Cassano GB (2005) Clinical correlates and significance of separation anxiety in patients with Bipolar Disorder. Bipol Disord 7: 370-376.
13. Klein DF (1993) False suffocation alarms, spontaneous panics, and related conditions An integrative hypothesis. Arch Gen Psychiatry 50: 306-317.

14. Manicavasagar V, Silove D, Hadzi-Pavlovic D (1998) Subpopulations of early separation anxiety: Relevance to risk of adult anxiety disorders. $J$ Affect Disord 48 : 181-190

15. Axelson DA, Birmaher B. (2001) Relation between anxiety and depressive disorders in childhood and adolescence. Depress Anxiety 14: 67-78.

16. Brady EU, Kendall PC (1992) Comorbidity of Anxiety and Depression in Children and Adolescents. Psychol Bull 111: 244-255.

17. Hale III WW, Raaijmakers QAW, Muris P, Hoof A van, Meeus WHJ (2009) One factor or two parallel processes?: Comorbidity and development of adolescent anxiety and depressive disorder symptoms. J Child Psychol Psychiatry 50: 1218-1226.

18. Bogels SM, Knappe S, Clark LA (2013). Adult Separation Anxiety Disorder in DSM-5. Clin Psychol Review 33: 663-674.

19. Van Steensel FJA, Bogels SM (2014) An adult version of the screen for child anxiety related emotional disorders (SCARED-A). Neth J Psychol 68: 81-87.

20. American Psychiatric Association (2000) Diagnostic and Statistical Manual of Mental Disorders. 4th ed. text rev., Washington, DC, American Psychiatric Association.

21. Moller EL, Bogels SM (2016) The DSM-5-dimensional anxiety scales in a dutch nonclinical sample: psychometric properties including the adult separation anxiety disorder scale. Int J Meth Psych Res 25: 232-239.

22. Does AJW van der (2002) BDI-II-NL Manual. The Dutch version of the Beck Depression Inventory-2nd edition, Lisse, The Netherlands, Harcourt Test Publishers.

23. Dozois DJ, Dobson KS, Ahnberg JL (1998) A psychometric evaluation of the Beck Depression Inventory-II. Psychol Assessment 10: 83-89.

24. Little RJA (1988) A test of missing completely at random for multivariate data with missing values. J Am Stat Ass 83: 1198-1292.

25. IBM Corp. Released 2016. IBM SPSS Statistics for Windows, Version 24.0, Armonk, New York, IBM Corp.

26. Raaijmakers QAW (1999) Effectiveness of different missing data treatments in surveys with Likert-type data: Introducing the relative mean substitution approach. Educational Psychol Measurement 59: 725-748.

27. Muris P, Bodden D, Hale WW, Birmaher B, Mayer B (2007) SCARED-NL: Manual of the revised Dutch version of the Screen for Child Anxiety Related Emotional Disorders, Amsterdam, Boom Test Uitgevers.

28. Watson D, Clark LA (1984) Negative affectivity: The disposition to experience aversive emotional states. Psychol Bull 96: 465-490.

29. Schneier FR, Moskow DM, Choo TH, Galfalvy H, Campeas R, Sanchez-Lacay A, et al. (2017) A randomized controlled pilot trial of vilazodone for adult separation anxiety disorder. Depress Anxiety 34: 1085-1095.

30. Baldwin DS, Gordon R, Abelli M, Pini S (2016) The separation of adult separation anxiety disorder. CNS Spectrums 21: 289-294.

Copyright: (C2018 Hale WW III. This is an open-access article distributed under the terms of the Creative Commons Attribution License, which permits unrestricted use, distribution, and reproduction in any medium, provided the original author and source are credited. 